In der Rubrik „Literatur kompakt" werden die wichtigsten Originalarbeiten aus der internationalen Fachliteratur referiert.

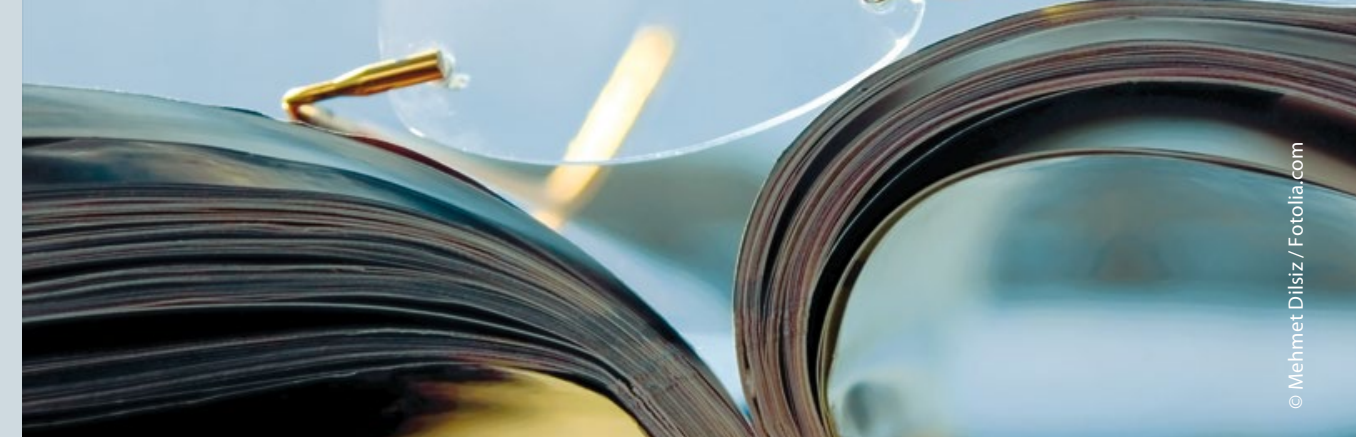

\section{Telemedizin ermöglicht Analgesie durch Notfallsanitäter}

\section{In einer Pilotstudie haben Aachener Notfallmediziner getestet, ob sich die prästationäre Schmerztherapie ohne Qualitätseinbußen an Rettungspersonal delegieren lässt. Ärzte unterstützten die Sanitäter über telemedizinische Kanäle.}

n der Schmerztherapie für Notfallpatienten legt der deutsche Gesetzgeber dem nicht ärztlichen Rettungspersonal Fesseln an. Speziell erlaubt das Betäubungsmittelgesetz nur Ärzten, Opioide zu verabreichen. Ist in einer Notfallsituation zunächst nur Rettungspersonal und kein Arzt anwesend, muss mit der entsprechenden Analgesie bis zum Eintreffen eines Mediziners gewartet wer-

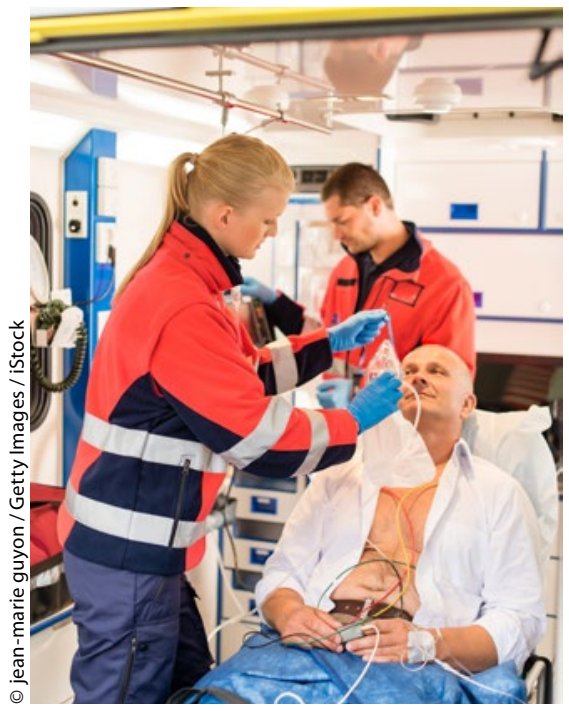

Durch eine Opioidgabe durch Rettungskräfte könnte die Zeit bis zum Eintreffen des Notarztes gespart werden. den, selbst wenn beispielsweise bereits ein intravenöser Zugang liegt.

Ärzte der Aachener Universitätsklinik, angeführt von Jörg Christian Brokmann, haben in einer Pilotstudie untersucht, ob sich diese Wartezeit mit dem Einsatz von Telemedizin vermeiden lässt. Sie rüsteten dafür fünf Sanitäterteams, die in vier nordrhein-westfälischen Rettungsbezirken (Euskirchen, Düren, Heinsberg und Aachen) eingesetzt wurden, mit einem multifunktionalen telemedizinischen System aus. Dadurch waren die Teams während des Einsatzes über verschiedene mobile Netze mit einem Notfallmediziner verbunden. Übertragen wurden die Vitaldaten der Patienten und Bilder oder ein Videostream vom Einsatzort und - nachdem der Patient transportfähig und geborgen war - aus dem Rettungsfahrzeug.

Die Wirksamkeit dieses Vorgehens wurde mit der von Einsätzen verglichen, die nach dem Normalschema verliefen. Bei denen also bis zum Eintreffen des Notarztes gewartet werden musste, bevor der Patient Opioide bekam. Verglichen wurden die Ergebnisse von 80 telemedizinisch unterstützten und 80 klassischen Einsätzen, in denen eine Schmerztherapie nötig war.

Im Ergebnis erwies sich die telemedizinisch gestützte Delegation der Analge- sie an das Rettungspersonal als sicher, die dadurch erzielte Schmerzreduktion war adäquat. Definitionsgemäß war dies gleichbedeutend mit einer Senkung des Schmerzgrades um mindestens zwei Punkte oder wenigstens auf unter fünf Punkte, gemessen mit einer numerischen Schmerzskala von 0 bis 10 (maximaler Schmerz). Dieses Ziel wurde in der Telemedizingruppe bei 61 von 65 vollständig dokumentierten Notfällen erreicht. In der Vergleichsgruppe gelang dies in 31 von 32 dokumentierten Fällen, die Differenz zur telemedizinische versorgten Gruppe war nicht signifikant ( $\mathrm{p}$ $=1,0)$. Im Mittel sanken die Schmerzen der telemedizinisch mitbetreuten Patienten um 3,8 und jene der in üblicher Weise Versorgten um 4,4 Punkte ( $\mathrm{p}=$ 0,02). 64 von 80 Patienten in der Telemedizingruppe und 74 von 80 Patienten in der Standardgruppe erhielten Opioide $(p=0,002)$. Bei jeweils 9 von 80 Patienten in beiden Gruppen traten Übelkeit und Erbrechen auf.

Fazit: Die Resultate der Pilotstudie zum Einsatz von Notfallsanitätern in der Schmerztherapie von Notfallpatienten sprechen dafür, die Verabreichung von Opioiden unter ärztlich-telemedizinischer Anleitung an Rettungspersonal zu delegieren. Die Schmerzreduktion übertraf in der Studie die Anforderungen. Zudem war unter den Bedingungen der Telemedizin die Qualität der Schmerzdokumentation mit $65 / 80$ versus $32 / 80$ ( $\mathrm{p}<0,0001)$ erheblich höher.

Brokmann JC et al. Analgesia by telemedically supported paramedics compared with physician-administered analgesia: A prospective, interventional, multicentre trial. Eur J Pain 2016; 20 : 1176-84 